\title{
Estilos de pensamiento y aprendizaje en estudiantes de magisterio y psicopedagogía: diferencias según curso y especialidad
}

\section{Thinking and learning styles among teaching and psychopedagogy students: differences according to course and speciality}

\author{
Patricia Robledo Ramón, Jesús Nicasio García Sánchez, Carmen Díez González, Mª Lourdes \\ Álvarez Fernández, Josefina Milagros Marbán Pérez, Ana Mª de Caso Fuentes, Raquel Fidalgo \\ Redondo, Olga Arias Gundín, Deilis Ivonne Pacheco Sanz
}

Universidad de León

Disponible online 30 de agosto de 2010

\begin{abstract}
Se presenta un estudio, inmerso dentro de un proyecto de innovación que se está desarrollando en la Universidad de León, orientado a adaptar distintas titulaciones psicoeducativas a las directrices del Espacio Europeo de Educación Superior partiendo para ello, en una primera fase del mismo, del análisis caracterológico del alumnado matriculado en la Facultad de Educación. Las características propias de los estudiantes, tales como sus estilos de pensamiento y sus enfoques de aprendizaje, pueden determinar el éxito del método de enseñanza-aprendizaje aplicado en el aula, por lo que conocer estos aspectos resulta indispensable como punto de partida previo a la introducción de innovaciones metodológicas. Por ello, el objetivo de este trabajo ha sido analizar estas variables psicológicas en una muestra de 583 alumnos de los diferentes cursos y especialidades de Magisterio y Psicopedagogía previamente a la implementación de metodologías activas en diversas asignaturas. Para evaluar estos aspectos se han utilizado el Cuestionario de Evaluación de los Procesos de Estudio y Aprendizaje y el Inventario de Estilos de Pensamiento. Los resultados señalan diferencias estadísticamente significativas en las variables evaluadas en relación a la especialidad y el curso de los alumnos, lo cual debe orientar a los docentes a la hora de seleccionar sus métodos didácticos, considerando las particularidades del alumnado.
\end{abstract}

Palabras clave: Estilos de pensamiento; Enfoques de aprendizaje; Innovación metodológica; Espacio Europeo de Educación Superior.

We present a study which forms part of an innovation project under development at the University of León, Spain, for adapting different psychoeducational degrees to the guidelines of the European Higher Education Area (EHEA). To this end, we first analysed the characteristics of the students enrolled in the Faculty of Education. The characteristics of the students, such as their thinking style and learning approach, may offer insight into the success of teaching-learning in the classroom; these aspects should be understood before introducing such innovative methodologies. Thus, these psychological variables were analysed in a sample of 583 students enrolled in different teaching and psycho-educational courses and specialities prior to the development of methodological innovations within diverse subject areas. We evaluated these aspects using the Questionnaire of Evaluation of the Processes of Study and Learning and the Thinking Styles Inventory. There were statistically significant differences between the variables relating to the students' speciality and course. These results should help to guide teachers when selecting the type of innovative methodologies to use in their classes in order to successfully adapt them to the specific characteristics of the students.

Key words: Thinking styles; Learning approaches; Innovative methodology; European Higher Education Area.

Este trabajo ha sido financiado por el Ministerio de Ciencia e Innovación de España (EDU2010-19250), con fondos FEDER de la Unión Europea para el trienio 2010-2013; así como del proyecto al Grupo de Investigación de Excelencia de la JCyL GR-259; (BOCyL 27 de abril de 2009) para el trienio 2009-2011, y ayudas competitivas de los proyectos PAID_ULE 2010. Dos becas de investigación pre-doctoral de la Junta de Castilla y León (JCyL), ayuda destinada a financiar la contratación de personal investigador de reciente titulación de la JCyL, así como becas FPI adscritas a proyectos MEC / MICINN.

Correspondencia: Jesús Nicasio García Sánchez, Universidad de León, Departamento de Psicología, Sociología y Filosofía, Área de Psicología Evolutiva y de la Educación, Campus de Vegazana, s/n, 24071-León, España, tf.: +34-987 29 10 41; +34-987 2931 58; móvil: +34-652 817871. E-mail: jn.garcia@unileon.es 
PATRICIA ROBLEDO RAMÓN, JESÚS NICASIO GARCÍA SÁNCHEZ, CARMEN DÍEZ GONZÁLEZ, Ma LOURDES ÁLVAREZ FERNÁNDEZ,

JOSEFINA MILAGROS MARBÁN PÉREZ, ANA Mª DE CASO FUENTES, RAQUEL FIDALGO REDONDO, OLGA ARIAS GUNDÍN, DEILIS IVONNE PACHECO SANZ

Actualmente en España la educación universitaria, inmersa en el marco del Espacio Europeo de Educación Superior (EEES), se encuentra asimilando progresivamente el cambio conceptual emergente y realizando ajustes importantes en su metodología pedagógica. Los nuevos planteamientos educativos en la Universidad se basan principalmente en concebir el aprendizaje como un proceso autónomo, presente a lo largo de toda la vida de la persona y que tiene como principal agente protagonista del mismo al estudiante. Se asume por tanto un nuevo paradigma de aprendizaje en el que el estudiante debe adquirir una mayor responsabilidad y un mayor auto-control en el proceso de construcción del mismo, mientras que el docente debe mutar su rol hacia la figura de guía o facilitador en el proceso educativo (Arias-Gundín, Fidalgo, y García, 2008). Se persigue con ello capacitar a los graduados para que, a través de sus estudios, sean capaces de adquirir todo un espectro de atributos explícitos que les permitan comprometerse eficazmente en el mundo laboral (Rodríguez, 2005), así como potenciar en ellos el desarrollo de competencias, tanto genéricas (instrumentales, personales y sistémicas) como específicas (Díez, et al., 2009; Fidalgo y García, 2008; Martínez-Cocó et al., 2008). Es decir, la universidad europea tiende hoy en día a estimular la autonomía y protagonismo del estudiante (Baños y Pérez, 2005), introduciendo para ello en las aulas innovaciones de carácter estructural y funcional, destacando entre ellas las de tipo metodológico.

En este sentido emerge con fuerza en el ámbito propiamente didáctico la propuesta de incluir en las aulas el uso de las llamadas metodologías activas, fundamentadas precisamente en estimular la autonomía y responsabilidad del estudiante a la hora de aprender (Gómez, García, y Villalba, 2005). Antes de la realización de cambios metodológicos drásticos es necesario conocer las características psicológicas de la población de estudiantes a los que se les va a requerir que trabajen con ese nuevo modo de proceder, ya que la forma en que las personas llevan a cabo las funciones relacionadas con el aprendizaje está asociada, por una parte, con fuentes externas, como pueden ser las estrategias o métodos de enseñanza y, por otra, con variables internas determinantes (English, Luckett, y Mladenovic, 2004; Struyven, Dochy, y Janssens, 2005). Desde esta perspectiva existen ciertos estudios selectivos (correlacionales) o incluso causales que ponen en relación el éxito escolar y variables motivacionales y cognitivas del alumnado (Bernardo, et al. 2009; Stenberg, Castejón, y Bermejo, 1999; Zhang, 2004; Zhang y Sternberg, 2005).

En relación con las primeras, existen importantes diferencias en la forma en que los estudiantes llevan a cabo las diversas funciones del aprendizaje, así como en el uso que hacen de los recursos instruccionales, dependiendo en todo momento de su estilo de aprendizaje. Igualmente se ha comprobado que los alumnos tienen distintas motivaciones y preferencias hacia determinadas estrategias cognitivas que les permiten con mayor facilidad dar significado a la nueva información (Demirbas y
Demirkan, 2007). Así, a lo largo de los años de investigación sobre el rendimiento y los factores determinantes del mismo, uno de los aspectos más estudiados ha sido el de los enfoques o estilos de aprendizaje (Demirbas y Demirkan, 2007; Li, Chen, y Tsai, 2008).

Los estilos de aprendizaje de las personas están asociados a unos determinados patrones motivacionales y atribucionales que definen su enfoque de aprendizaje. Diseñar estrategias de enseñanza que ayuden al alumnado a superar las debilidades y aprovechar las fortalezas de estos aspectos, contribuirá a obtener mejoras en el rendimiento académico, motivación, actitudes y desarrollo de habilidades (Farkas, 2003). En relación con las innovaciones metodológicas que emergen, varias investigaciones evidencian que el uso de diferentes procedimientos metodológicos tiene como consecuencia una instrucción más efectiva, sobre todo cuando se tiene en consideración el estilo de aprendizaje del alumno (Felder, Felder, y Dietz, 2002; Rayner y Riding, 1997); por lo tanto, dependiendo del grado en que la orientación instruccional encaje con el modo de aprender de los estudiantes, éstos se verán más o menos favorecidos y tendrán una mayor o menor probabilidad de éxito (Araz y Sungur, 2007; Chapman y Calhoun, 2006; Hur y Kim, 2007; McDonough y Osterbrink, 2005). Por ello, previamente a la implementación de innovaciones metodológicas en las aulas, es necesario conocer estas dimensiones personales del alumnado, siendo este aspecto uno de los abordados en el presente trabajo.

En relación con las variables individuales de tipo cognitivo, la tendencia investigadora se ha trasladado progresivamente desde perspectivas centradas exclusivamente en análisis de la aptitud o inteligencia, hacia corrientes focalizadas en estudiar los estilos de pensamiento y su papel en el proceso de enseñanza-aprendizaje (González-Pienda, et al., 2004). El estilo de pensamiento, definido como la forma predilecta que tiene la persona de pensar (Stenber y Grigorenko, 1995), se relaciona con la capacidad del alumno para dirigir su propia cognición y consecuentemente su propio proceso de aprendizaje, por lo que, la propia definición del constructo sustenta su conexión con los parámetros autónomos de aprendizaje pretendidos por los nuevos principios rectores de la enseñanza superior. El estudio de este constructo psicológico parte principalmente de las aportaciones realizada por Stenberg, quien aborda su análisis en la denominada Teoría del autogobierno mental, desde la que se identifican 13 estilos de pensamiento agrupados en 5 dimensiones: función (legislativo, ejecutivo y judicial), forma (jerárquico, oligárquico, monárquico y anárquico), nivel (local y global), campo (interno y externo), y tendencia (conservador y liberal). A partir de aquí y a pesar de las nuevas aportaciones y avances en torno a este modelo (González-Pienda, et al., 2004) diversos trabajos sostienen que los estilos de pensamiento contribuyen significativamente a la explicación de la variabilidad en el rendimiento de los estudiantes de cualquier nivel escolar, incluyendo el nivel universitario (Bernardo, et al., 2009). Por lo tanto, dado que es necesario estimular el aprendizaje autónomo 
del alumno, fomentando el control que él mismo debe ejercer del propio proceso, parece obvia la necesidad de conocer los estilos de pensamiento del estudiante, ya que esto permitirá realizar una mejor adaptación de los métodos de enseñanza y asegurar un aprendizaje de más calidad.

Como consecuencia de lo expuesto, las prácticas universitarias deben estar centradas en la persona que aprende, considerando los procesos internos que pone en funcionamiento durante el aprendizaje. Por ello, antes de la introducción de cambios metodológicos sustanciales en las actividades docentes universitarias, es necesario considerar, tanto el enfoque de aprendizaje, como el estilo intelectual de los alumnos, ya que ambos aspectos pueden condicionar la eficacia de la metodología aplicada sobre el rendimiento, así como su potencial para promover el desarrollo de competencias (González, 2002; Martínez y Crespo, 2008; Salim, 2005). En este contexto es donde se incardina el presente trabajo, el cual forma parte de un proyecto de innovación mucho más amplio que se está desarrollando en la Universidad de León orientado precisamente a renovar la metodología universitaria con el fin de adaptar las diferentes especialidades de Magisterio y la titulación de Psicopedagogía a las nuevas directrices propuestas en el EEES (García et al, 2008). No obstante, como se ha señalado, antes de realizar este cambio conceptual y metodológico, es necesario conocer algunas de las características psicológicas de la población de estudiantes a los que se les va a instar a convertirse en agentes dinámicos en su propio proceso de aprendizaje, para asegurar, en la medida de lo posible, el éxito de la innovación metodológica. Así pues, el primer objetivo del presente estudio ha sido identificar los estilos de pensamiento, estrategias, motivaciones y enfoques de aprendizaje del alumnado matriculado en las dos titulaciones, Magisterio y Psicopedagogía, impartidas en la Facultad de Educación de la Universidad de León (ULE), previamente a la introducción de métodos activos en varias de las materias que éste debía cursar de manera obligatoria. Dado que las titulaciones constan de varios cursos y están compuestas por diferentes especialidades, en las cuales se abordan temáticas muy diversas, tienen unos requisitos de acceso diferentes en cuanto a notas de corte o formación previa y dan lugar a perfiles profesionales muy dispares, resulta de interés controlar además las variables curso y especialidad en relación a los factores analizados (estilos de pensamiento y aprendizaje).

Han sido escasos los estudios realizados en España que analicen las variaciones posibles de estas variables psicológicas en relación a las dimensiones curso o especialidad, sobre todo si nos ceñimos de manera específica a las titulaciones de Magisterio y Psicopedagogía y, además, los datos no han sido concluyentes. Por una parte, en el trabajo realizado por Gil et al. (2007) se concluye que los estilos de aprendizaje van modificándose a medida que los alumnos pasan de un curso a otro, incrementándose en este sentido los estilos más adaptativos. En cambio, en otro estudio específico de Magisterio en el que sólo se consideró la variable curso en la especialidad de Educación
Física no se encontraron diferencias en los estilos de aprendizaje en función del curso (Gómez, 2003). Sin embargo, la tendencia parece indicar que existen diferencias en los estilos de aprendizaje de los alumnos en función de su curso comparando varias titulaciones, aunque estas no sean de la misma rama. Los trabajos en este sentido señalan la variación con el curso hacia unos enfoques más adecuados, señalando la importancia de esta variable para comprender el comportamiento estratégico del alumno (Cano y Justicia, 2003). Además, estos resultados parecen observarse también en las investigaciones centradas en los estilos de pensamiento, obteniéndose diferencias en función de las titulaciones (González, Castro, y González, 2008), aunque no se conocen estudios que aborden las titulaciones de interés de este trabajo. Por todo ello, se plantea un segundo objetivo de estudio centrado en averiguar las diferencias por curso y especialidad en los dos constructos psicológicos de interés (estilos de pensamiento y estrategias de aprendizaje).

\section{Método}

\section{Participantes}

La muestra de alumnos participantes en el estudio estuvo compuesta por un total de 583 estudiantes matriculados en siete especialidades diferentes de la Diplomatura de Magisterio y en la titulación de segundo ciclo, Psicopedagogía, de la ULE (Ver para más detalle Tabla 1).

Tabla 1. Distribución de participantes en relación a la especialidad que cursan y su sexo.

\begin{tabular}{lccc}
\hline Especialidad & Hombre & Mujer & Total especialidad \\
\hline Audición y Lenguaje & 1 & 16 & 17 \\
Educación Especial & 4 & 69 & 73 \\
Educación Física & 92 & 41 & 133 \\
Educación Infantil & 4 & 82 & 86 \\
Educación Musical & 16 & 32 & 48 \\
Educación Primaria & 26 & 71 & 97 \\
Lengua Extranjera & 10 & 29 & 39 \\
Psicopedagogía & 15 & 66 & 81 \\
\hline Total sexo & 168 & 406 & 574
\end{tabular}

Nota: Si bien el total de participantes fueron $N=583$, únicamente se incluyen en cada variable de agrupamiento aquellos alumnos de los que se disponen datos.

Dichos estudiantes pertenecían a diferentes cursos, concretamente a $1^{\circ}(n=239), 2^{\circ}(n=167)$ y $3^{\circ}(n=65)$ de Magisterio y $4^{\circ}(n=46)$ y $5^{\circ}(n=29)$ de Psicopedagogía; sus edades oscilaban entre los 17 años de los alumnos de primero y los 40 años de alguno de los estudiantes de tercer curso, siendo la media de edad de la muestra de 20.80 años.

Todos los participantes tenían como característica común que iban a cursar una o varias asignaturas implementadas de 
PATRICIA ROBLEDO RAMÓN, JESÚS NICASIO GARCÍA SÁNCHEZ, CARMEN DÍEZ GONZÁLEZ, Ma LOURDES ÁLVAREZ FERNÁNDEZ, JOSEFINA MILAGROS MARBÁN PÉREZ, ANA Mª DE CASO FUENTES, RAQUEL FIDALGO REDONDO, OLGA ARIAS GUNDÍN, DEILIS IVONNE PACHECO SANZ

manera innovadora por profesorado perteneciente al área de Psicología Evolutiva y de la Educación. Concretamente, las materias en las que se implementaron las innovaciones metodológicas fueron: Bases Psicológicas de la Educación Especial, Aspectos Evolutivos de la Deficiencia Motórica, Psicología de la Educación y del Desarrollo en la Edad Escolar, Aprendizaje y Desarrollo Motor, Psicología Infantil, Educación Afectivo sexual, Procesos Psicológicos Básicos, Dificultades de Aprendizaje e Intervención psicopedagógica e Intervención Psicopedagógica en los Trastornos del Desarrollo. En cuanto a las metodologías activas empleadas por los profesores destacan el Aprendizaje Basado en Problemas (Fernández, García, de Caso, Fidalgo y Arias, 2006; García, 2002; García, de Caso, Fidalgo y Arias, 2005) y el Estudio de Caso (Arias et al., 2008), y/o herramientas innovadoras como el Portafolio del alumno y/o las plataformas digitales Aulaunileon (García et al., 2008), Blogs educativos (Robledo, Marbán, y García, 2008) y Moodle (https://www3.unileon.es/personal/wwjngars/moodle). Antes de la implementación del plan de innovación-investigación los universitarios fueron informados de los objetivos del mismo e invitados a participar, de manera voluntaria, respondiendo a las cuestiones que se les plantearon mediante los diferentes instrumentos de evaluación utilizados. Se siguieron las normas éticas y deontológicas exigidas en toda investigación científica.

\section{Instrumentos}

Los instrumentos de medida utilizados para analizar las variables objeto de estudio fueron dos test estandarizados, comercializados y validados empíricamente en castellano y ampliamente utilizados a nivel nacional e internacional, elaborados por expertos. En primer lugar, fruto de una adaptación del Study Process Questionnaire (SPQ) de Biggs, se utilizó el Cuestionario de Evaluación de los Procesos de Estudio y Aprendizaje (CEPEA) (Barca, Seijas, Brenlla y Santamaría, 2000), instrumento que presenta unas propiedades psicométricas adecuadas, oscilando el índice de fiabilidad por consistencia interna, utilizando el $\alpha$ de Cronbach, entre .76 y .79. El CEPEA consta de 42 ítems que evalúan las motivaciones, estrategias y enfoques de aprendizaje que adoptan los estudiantes universitarios en sus procesos de estudio. Por otra parte, se aplicó la adaptación realizada por González-Pienda y colaboradores (1997) del Inventario de Estilos de Pensamiento (MSG) (Sternberg y Wagner, 1991), instrumento que permite conocer los estilos intelectuales de los alumnos en cinco dimensiones: funciones, ámbitos, formas, niveles y tendencias. En la Tabla 2 se presentan los cuestionarios de manera más detallada, especificando las subescalas que componen cada uno de ellos y los constructos psicológicos evaluados.

Tabla 2. Descripción del Cuestionario de Evaluación de Procesos de Estudio y Aprendizaje (CEPEA) y del Inventario de Estilos de Pensamiento (MSG).

\begin{tabular}{|c|c|}
\hline Nombre & Subescalas: aspectos evaluados \\
\hline $\begin{array}{l}\text { Cuestionario de Evaluación de Procesos de Estudio } \\
\text { y Aprendizaje (CEPEA) }\end{array}$ & $\begin{array}{l}\text { Enfoque de aprendizaje profundo }(\mathrm{EP})=\text { Motivación profunda }(\mathrm{MP})+\text { estrategia profunda (EsP). } \\
\text { Enfoque de aprendizaje superficial }(\mathrm{ES})=\text { Motivación superficial }(\mathrm{MS})+\text { estrategia superficial (EsS). } \\
\text { Enfoque de aprendizaje de logro }(\mathrm{EL})=\text { Motivación logro }(\mathrm{ML})+\text { estrategia logro (EsL). } \\
\text { Compuesto enfoque profundo + enfoque logro (CP-L). } \\
\text { Compuesto enfoque superficial + enfoque logro (CS-L). }\end{array}$ \\
\hline Inventario de Estilos de Pensamiento (MSG) & $\begin{array}{l}\text { Trece estilos intelectuales agrupados en cinco dimensiones: } \\
\text { Funciones: legislativo, judicial y ejecutivo. } \\
\text { Formas: monárquico, jerárquico, oligárquico y anárquico. } \\
\text { Niveles: global y local. } \\
\text { Ámbito: interno y externo. } \\
\text { Tendencias: conservador y liberal. }\end{array}$ \\
\hline
\end{tabular}

\section{Procedimiento}

El presente estudio se llevó a cabo en la Facultad de Educación de la ULE, durante el curso académico 2007-2008. Para su desarrollo, previamente al inicio de las clases y al desarrollo de las asignaturas dependientes del área de Psicología Evolutiva y de la Educación mediante métodos innovadores, diferentes miembros del equipo aplicaron de manera grupal las escalas de evaluación presentadas a la muestra de alumnos descrita anteriormente y que, de manera voluntaria, se prestó a colaborar en el estudio.

Concluido el trabajo de campo y la recogida de los protocolos cumplimentados por los estudiantes, se llevó a cabo la informatización y codificación en una matriz datos para la realización de los análisis estadísticos oportunos, mediante SPSS versión 13.0, que han aportado las evidencias empíricas de la investigación.

\section{Resultados}

El tipo de análisis estadístico realizado ha sido no paramétrico puesto que las pruebas de asimetría y curtosis realizadas inicialmente determinaron que la distribución no cumplía los supuestos de normalidad en varias de las variables analizadas. Por ello, y con la finalidad de evidenciar las diferencias existentes en las medidas estudiadas, se utilizó la prueba de Kruskall-Wallis. En dichos análisis se tomaron como variables 
de agrupamiento, por una parte, el curso de los alumnos $\left(1^{\circ}, 2^{\circ}\right.$ y $3^{\circ}$ de Magisterio y $4^{\circ}, 5^{\circ}$ de Psicopedagogía) y, por la otra, su especialidad y se relacionaron con las diferentes medidas obtenidas a través del CEPEA y del MSG, obteniéndose resultados significativos en la mayoría de ellas.

Comenzando por los resultados obtenidos al considerar el curso como factor fijo, en la Tabla 3 se presentan de manera detallada las diferencias estadísticamente significativas encontradas en las variables psicológicas de los estudiantes.
Atendiendo en primer lugar a los datos obtenidos a través del cuestionario CEPEA y centrándonos exclusivamente en las dimensiones Motivación y Estrategias de aprendizaje, se puede observar que los alumnos de $1^{\circ}$ y $2^{\circ}$ de Magisterio presentan más superficialidad $(p=.001)$ y orientación hacia el logro $(p=.001)$ en estos dos aspectos. En cambio, los estudiantes de los últimos cursos de carrera, ya sean los de $3^{\circ}$ de la diplomatura de Magisterio o los de $5^{\circ}$ de la licenciatura de Psicopedagogía, se caracterizan por tener unas motiva-

Tabla 3. Diferencias en las escalas del Cuestionario de Evaluación de Procesos de Estudio y Aprendizaje (CEPEA) y del Inventario de Estilos de Pensamiento (MSG) según curso.

\begin{tabular}{|c|c|c|c|c|c|c|c|}
\hline \multirow[t]{2}{*}{ Escalas/Variables } & \multicolumn{5}{|c|}{ Curso } & \multirow[t]{2}{*}{$\chi^{2}$} & \multirow[t]{2}{*}{$p$} \\
\hline & $1^{\mathrm{o}}$ & $2^{\circ}$ & $3^{\circ}$ & $4^{\circ}$ & $5^{\circ}$ & & \\
\hline \multicolumn{8}{|c|}{ Cuestionario de evaluación de procesos de estudio y aprendizaje (CEPEA) } \\
\hline Motivación profunda & 240.90 & 185.60 & 245.70 & 214.60 & 221.20 & 18.00 & .001 \\
\hline Motivación logro & 253.70 & 190.90 & 217.50 & 171.20 & 190.40 & 24.60 & .001 \\
\hline Estrategia superficial & 223.00 & 235.80 & 232.10 & 156.20 & 148.40 & 14.20 & .006 \\
\hline Estrategia profunda & 220.30 & 196.40 & 251.60 & 254.60 & 284.60 & 15.80 & .003 \\
\hline Estrategia logro & 248.00 & 184.30 & 215.80 & 230.60 & 241.00 & 21.10 & .001 \\
\hline Enfoque superficial & 241.00 & 216.10 & 214.70 & 162.00 & 159.60 & 14.00 & .007 \\
\hline Enfoque profundo & 232.90 & 185,10 & 250.50 & 238.50 & 261.70 & 18.80 & .001 \\
\hline Enfoque logro & 258.50 & 178.70 & 215.40 & 192.90 & 214.10 & 33.40 & .001 \\
\hline \multicolumn{8}{|c|}{ Inventario de Estilos de Pensamiento (MSG) } \\
\hline Estilo Anárquico & 230.40 & 197.90 & 235.50 & 200.90 & 273.80 & 10.80 & .028 \\
\hline Estilo Interno & 237.90 & 211.90 & 226.50 & 145.30 & 190.80 & 11.40 & .022 \\
\hline
\end{tabular}

Nota: AL: Audición y Lenguaje; EE: Educación Especial; EF: Educación Física; EI: Educación Infantil; EM: Educación Musical; EP: Educación Primaria; LE: Lengua Extranjera; PS: Psicopedagogía.

ciones $(p=.001)$ y utilizar unas estrategias de aprendizaje $(p$ $=.003)$ de carácter más profundo o, al menos, como en el caso de los alumnos de $4^{\circ}$, menos superficiales. Atendiendo ahora a la dimensión Enfoques de aprendizaje, la cual se obtiene sumando las variables motivación y estrategias, las puntuaciones indican cómo aquellos universitarios matriculados en una titulación de segundo ciclo, especialmente los del último año $\left(5^{\circ}\right)$, seguidos de los del último curso de Magisterio $\left(3^{\circ}\right)$, son los que presentan unos enfoques de aprendizaje más profundos $(p=.001)$. En el polo opuesto, los estudiantes cuyo enfoque es más superficial son los del primer curso $(p=.007)$, quienes también muestran una clara orientación hacia el logro $(p=.001)$.

Considerando en segundo lugar los estilos de pensamiento de los alumnos, evaluados a través del Inventario de estilos de pensamiento (MSG), en relación con su curso, se han encontrado igualmente varias diferencias estadísticamente significativas. Así, en el estilo Local los alumnos de $3^{\circ}$ (Rango Medio $=261$ ) son los que puntúan más alto, mientras que los de $2^{\circ}$ $($ Rango Medio $=195)$ obtienen las menores puntuaciones. Lo mismo sucede con el estilo Anárquico, aunque en este caso los que presentan mayores puntuaciones son los de quinto (Rango
Medio $=273)$ en comparación con los de segundo curso (Rango Medio $=197)$. Finalmente, los datos señalan que los alumnos de los primeros cursos $\left(1^{\circ}, 2^{\circ}\right.$ y $\left.3^{\circ}\right)$ obtienen mayores puntuaciones en estilo Interno que aquellos que cursan estudios de segundo ciclo (ej. $1^{\circ}$ Magisterio, Rango Medio $=237$ vs. $4^{\circ}$ Psicopedagogía, Rango Medio = 145).

Al considerar la especialidad como variable de agrupamiento y centrándonos exclusivamente de nuevo en el CEPEA, concretamente en las dimensiones Motivación y Estrategias de aprendizaje, los datos señalan que los alumnos de Audición y Lenguaje (Rango Medio $=291.60)$ presentan más profundidad en estos dos aspectos. Por el contrario, los estudiantes de Educación Física muestran menos profundidad en ambas dimensiones, lo cual no indica que sean los que destacan por la superficialidad máxima ( $p=.001)$, siendo los estudiantes de Lengua Extranjera los que muestran unas motivaciones más superficiales (Rango Medio = 267) y los de Educación Infantil los que presentan más superficialidad en sus estrategias (Rango Medio = 264). Por último, los alumnos perteneciente a la especialidad de Educación Primaria son los universitarios que presentan unas estrategias de aprendizaje más orientadas hacia el logro $($ Rango Medio $=265)$. 
PATRICIA ROBLEDO RAMÓN, JESÚS NICASIO GARCÍA SÁNCHEZ, CARMEN DÍEZ GONZÁLEZ, Ma LOURDES ÁLVAREZ FERNÁNDEZ, JOSEFINA MILAGROS MARBÁN PÉREZ, ANA Mª DE CASO FUENTES, RAQUEL FIDALGO REDONDO, OLGA ARIAS GUNDÍN, DEILIS IVONNE PACHECO SANZ

Figura 1. Diferencias en rango medios en la variable Enfoques de Aprendizaje del cuestionario CEPEA en función de la especialidad de los alumnos.

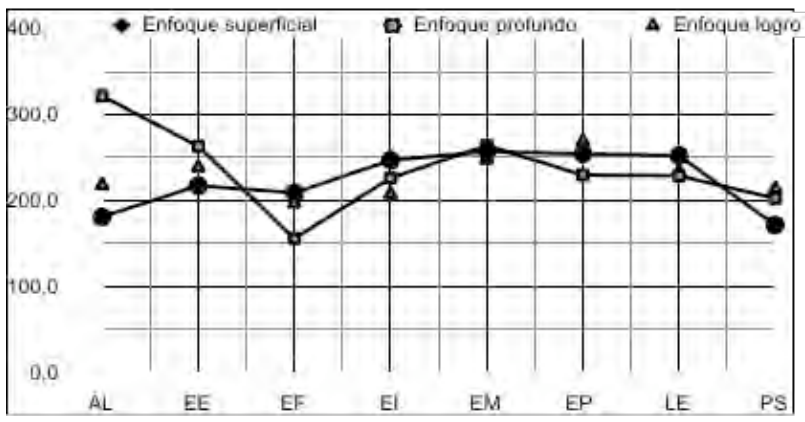

Nota: AL: Audición y Lenguaje; EE: Educación Especial; EF: Educación Física; EI: Educación Infantil; EM: Educación Musical; EP: Educación Primaria; LE: Lengua Extranjera; PS: Psicopedagogía.

En relación a la dimensión Enfoques de aprendizaje, la cual se obtiene sumando las variables motivación y estrategias, como se observa en la Figura 1, los alumnos de Audición y Lenguaje son los que presentan unos enfoques de aprendizaje más profundos, lo cual era de esperar dado que eran los que puntuaban más alto en las dos variables que constituyen esta dimensión. En el polo opuesto, los estudiantes cuyo enfoque es más superficial son los de la especialidad de Educación Musical $(p=.005)$. Por último, los alumnos de Educación Primaria son los que muestran un enfoque de aprendizaje más orientado hacia el logro $(p=.013)$. Los datos indican también que los alumnos de la especialidad de Audición y Lenguaje son los que obtienen puntuaciones más altas en el compuesto profundologro (Rango Medio $=278$ ), mientras que aquellos que están matriculados en Educación Primaria lo hacen en el compuesto totalmente opuesto, el denominado superficial-logro (Rango Medio $=268$ )

Figura 2. Diferencias en rangos medios de las escalas del Inventario de Estilos de Pensamiento en función de la especialidad cursada por los alumnos.

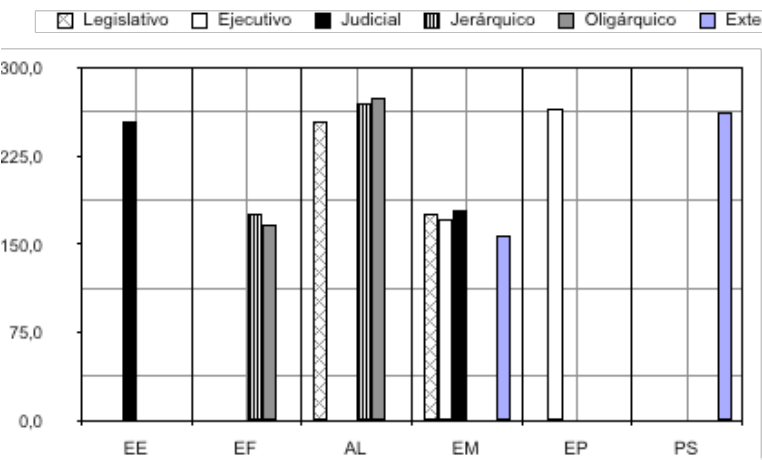

Nota: AL: Audición y Lenguaje; EE: Educación Especial; EF: Educación Física; EI: Educación Infantil; EM: Educación Musical; EP: Educación Primaria; LE: Lengua Extranjera; PS: Psicopedagogía.

Atendiendo a los estilos de pensamiento del alumnado (MSG) en relación a su especialidad se ha encontrado que en los estilos jerárquico y oligárquico los alumnos de Audición y Lenguaje son los que puntúan más alto, mientras que los de Educación Física obtienen las menores puntuaciones ( $p=.001)$. Lo mismo sucede con el estilo legislativo, aunque en este caso los que presentan menores puntuaciones son los de Educación Musical $(p=.033)$, quienes además, puntúan más bajo en los estilos externo, ejecutivo y judicial, como se detalla en la Figura 2.

\section{Discusión}

Muchas han sido las investigaciones que han evidenciado las diferencias existentes en las maneras de pensar y aprender de los estudiantes y que sostienen que la falta de atención a estas particularidades individuales puede derivar en inadaptaciones que afectan al rendimiento académico (Riding y Rayner, 2002; Tickle, 2001). Desde esta perspectiva, consideramos que un punto de partida básico, previo a la implementación de innovaciones metodológicas en la educación superior, debe ser conocer cómo aprenden los alumnos, cuáles son los estilos de aprendizaje predominantes entre ellos y sus estilos de pensamiento. Por ello, el objetivo de este estudio ha sido conocer, previamente a la inclusión de metodologías activas en diferentes asignaturas dependientes del área de Psicología Evolutiva y de la Educación, los enfoques de aprendizaje que utilizan los universitarios cuando se enfrentan a un nuevo curso académico, así como los estilos de pensamiento que les caracterizan, todo ello en relación a su curso y especialidad. Considerando los resultados obtenidos en el estudio se pueden extraer diversas conclusiones de interés, las cuales pueden contribuir a la hora de llevar a cabo innovaciones de tipo metodológico orientadas a adecuar progresivamente la situación de la educación superior española a las nuevas directrices europeas.

En primer lugar, según apuntan diferentes estudios (Baños, et al., 2005), los alumnos considerados óptimos para conseguir excelentes resultados académicos mediante la introducción de metodologías activas serían los que, por sus características, desarrollasen un enfoque de aprendizaje profundo ya que las personas que presentan este enfoque se caracterizan por tener unas motivaciones intrínsecas que les llevan a intentar comprender y satisfacer de manera reflexiva las diferentes materias, intentado realizar un aprendizaje significativo de las mismas mediante la conexión de los contenidos con sus conocimientos y experiencias previas. Además, este tipo de alumnos requerirían menor grado de estructura instruccional y menos apoyo de la enseñanza formal, por lo que representan el ideal de alumno activo propuesto por los nuevos modelos de Educación Superior. Los resultados de la investigación aquí presentada determinan que este tipo de estudiante es el que, al parecer, representan los universitarios de los últimos cursos de carrera, ya sean los de $3^{\circ}$ de Magisterio o bien los que cursan la titulación de $2^{\circ}$ ciclo Psicopedagogía. Estos datos coinciden con los aportados por García (2005) quien corroboró que a mayor edad en los estudi- 
antes mayor incremento de los enfoques de aprendizaje de tipo profundo, posiblemente debido a que la experiencia como aprendices contribuye a que las personas activen formas de aprender más adecuadas y propias de los aprendices expertos (Justicia, 2000). Sin embargo, este resultado no es concluyente, porque otros estudios, como el desarrollado por Martín y Camarero (2001), no evidencian diferencias, ni en relación al curso, ni a la especialidad. Por ello, son necesarios más trabajos en este ámbito que clarifiquen estas relaciones, si bien, los resultados más coincidentes apuntan una tendencia similar a la obtenida en nuestro presente estudio, refiriéndose a un mayor uso de estrategias adecuadas y un procesamiento más profundo en los alumnos de cursos finales (Camarero, Martín, y Herrero, 2000).

En cuanto a la especialidad, los universitarios de Audición y Lenguaje parecen ser los que se muestran más profundamente motivados hacia la carrera, denotando unas estrategias de aprendizaje más profundas. En el polo opuesto se encontrarían aquellos alumnos interesados esencialmente en evitar el fracaso, cumpliendo los requisitos mínimos exigidos por la tarea para aprobar y cuya estrategia de aprendizaje se basaría en la memorización y reproducción de lo aprendido. Este grupo estaría encabezado por los alumnos de $1^{\circ}$ y $2^{\circ}$ curso o, si se considera la especialidad, los de Educación Musical. Una posible explicación a este resultado se relaciona con los hallazgos de otros estudios en los que se evidenció que los alumnos de Educación Musical suelen presentar un mayor interés por los contenidos relacionados con las propias actividades musicales que por aquéllos ligados a la labor docente, mostrando en muchas ocasiones desinterés por ciertas materias de carácter didáctico (Camina y Salvado, 2007) y explicándose de este modo la escasa motivación presentada hacia ellas. Es decir, se trataría de estudiantes con una vocación más orientada a la propia música que a la enseñanza de la misma, por lo que sus motivaciones hacia la titulación serían ciertamente superfluas. Se da la circunstancia de que se trata de los alumnos de los primeros cursos, pudiéndose explicar esta baja motivación por el hecho de que el acceso a la especialidad haya sido "accidental" al no poder optar, por falta de la calificación necesaria, a la titulación deseada (Camina, et al., 2007), decidiéndose en este caso por Magisterio dadas las notas de corte menos elevadas para cursar esta titulación exigidas la ULE. Además, especialmente los de $1^{\circ} \mathrm{o}$ los de Educación Primaria tienden a desarrollar un enfoque, estilo y motivación de aprendizaje orientado hacia el logro de calificaciones académicas altas, motivados por sobresalir y que rentabilizan sus esfuerzos al máximo. Es probable que este resultado se relacione, por una parte, con la vocación de estos alumnos que se deciden por una especialidad pura de maestros y que, por ello, muestran interés en sacar calificaciones elevadas que les ayuden a tener éxito en las oposiciones y poder desempeñar la profesión que vocacionalmente desean (Bertomeu, et al, 1999); por otra parte, puede derivar de los estudios previos de estos alumnos, ya que con frecuencia los universitarios que acceden a magisterio provienen de Bachillerato (Camina et al,
2007) y, por lo tanto, han llegado presionados por la necesidad de obtener buenas calificaciones para superar la selectividad; no obstante, estas son unas aseveraciones que es preciso comprobar en estudios posteriores. A pesar de todo, este alumnado, con enfoques de aprendizaje menos adecuados, puede obtener resultados satisfactorios con las metodologías activas siempre y cuando reciban apoyo instruccional continuado por parte del profesor y reconocimiento frecuente de los éxitos obtenidos (Barca et al., 2000; de la Fuente, 2002).

En cuanto a los estilos de pensamiento de los alumnos, evaluados a través del MSG, se ha encontrado que, dependiendo del curso en el que estén, éstos tienden a caracterizarse por unos estilos de pensamiento concretos que se deberían considerar a la hora de instaurar métodos activos. Así, las diferencias se han dado en primer lugar en cuanto a las formas de dirigir el pensamiento. Destaca que los estudiantes de $5^{\circ}$ curso desarrollan una forma predominantemente anárquica en relación con el resto de universitarios de los otros cursos, mostrando motivación por más de un objetivo. Estos resultados están en consonancia con los encontrados por González, Castro y González (2008), quienes identificaron que los estudiantes de titulaciones relacionadas con la Psicología muestran un estilo ejecutivo en el que predomina la forma anárquica, caracterizada por la tendencia a evitar reglas, normas o procedimientos típicos, a la hora de abordar las tareas o los problemas. Esta característica, a pesar de aparentar cierta negatividad, se pueden tornar en un aspecto positivo si se tiene en cuenta que estos egresados, como psicopedagogos, se tendrán que enfrentar a una profesión cargada de problemáticas personales muy diversas a las que deberán dar respuestas dispares. Además, Sternberg y Grigorenko (1995) afirman que los alumnos con un estilo de pensamiento anárquico tienen problemas para adaptarse a un sistema formal de enseñanza, lo cual, a pesar de parecer una limitación, de nuevo podría ser positivo, ya que el nuevo modelo que emerge propone métodos de enseñanza menos rígidos y pautados que no exigirían a los universitarios ajustarse a unos patrones de enseñanza altamente dirigidos. Si bien esta es una hipótesis que es necesario testar en estudios posteriores. En cuanto a la tendencia de los alumnos a relacionarse con los demás a la hora de trabajar, los alumnos de $1^{\circ}$ se muestran más introvertidos, centrados en el trabajo y con una pequeña conciencia social, posiblemente debido a la falta de confianza en el grupo; en cambio, los de Psicopedagogía están mas centrados en las personas y con una gran conciencia social, lo cual les beneficiará para su desarrollo profesional posterior, en el que tendrán que estar en contacto y trabajar en cooperación con otros profesionales para ayudar a sus alumnos (García, 2005). Por último, los de $3^{\circ}$, a diferencia del resto, son los que prefieren en mayor medida trabajar con detalles centrados en cuestiones concretas, desarrollando un estilo de pensamiento local.

Atendiendo a este mismo constructo en relación a la especialidad cursada por los estudiantes, se puede concluir que, dependiendo de ésta, los universitarios tienden a caracterizarse 
PATRICIA ROBLEDO RAMÓN, JESÚS NICASIO GARCÍA SÁNCHEZ, CARMEN DÍEZ GONZÁLEZ, Ma LOURDES ÁLVAREZ FERNÁNDEZ, JOSEFINA MILAGROS MARBÁN PÉREZ, ANA Mª DE CASO FUENTES, RAQUEL FIDALGO REDONDO, OLGA ARIAS GUNDÍN, DEILIS IVONNE PACHECO SANZ

por unos estilos de pensamiento específicos. Así, en relación con las funciones de gobierno del pensamiento, hay diferencias entre alumnos legislativos (Audición y Lenguaje) que prefieren las actividades creativas, estudiantes ejecutivos (Educación Primaria) quienes se inclinan por realizar tareas o finalmente, los de carácter judicial (Educación Especial) los cuales prefieren analizar información y dar opiniones. Esto se podría relacionar con los propios intereses que han llevado a los alumnos a optar por estas especialidades y con las competencias que tendrán que desarrollar como futuros especialistas y que definen su perfil profesional según determina el Libro Blanco de Magisterio (ANECA, 2005), fundamento para establecer los nuevos grados. En cuanto a las formas de dirigir el pensamiento, destaca que los estudiantes de Audición y Lenguaje, aunque generalmente desarrollan una forma predominantemente oligárquica, mostrando motivación por varios objetivos cuya importancia es semejante, puntúan también alto en la forma jerárquica, lo que señala que se muestran motivados por más de una meta y establecen prioridades. Finalmente, en cuanto a la tendencia de los alumnos a relacionarse con los demás a la hora de trabajar, los alumnos de Psicopedagogía se muestran más extrovertidos, centrados en las personas y con una gran conciencia social, lo cual encaja con las demandas de su futuro profesional (García, 2005).

En definitiva, los resultados obtenidos en este estudio nos permiten concluir que los alumnos universitarios, dependiendo del curso en el que estén y la especialidad que eligen, presentan unos intereses y motivaciones diferentes hacia el aprendizaje, así como unos estilos de pensamiento específicos y delimitados que se deben considerar a la hora de trabajar con ellos de manera efectiva. Por lo tanto, previamente a la implantación de innovaciones docentes orientadas a adaptar los actuales títulos universitarios a los requisitos del nuevo EEES, es imprescindible obtener información acerca de las características psicológicas de los alumnos. Esto servirá al profesorado para inclinarse por el uso de unas herramientas metodológicas concretas a través de las cuales logrará una aproximación progresiva pero eficaz a los modelos propuestos a nivel europeo, especialmente en lo que se refiere a la adquisición de competencias.

\section{Referencias}

Agencia nacional de evaluación de la calidad y acreditación (2005). Libro Blanco título de Grado de Magisterio. Madrid: ANECA.

Araz, G. y Sungur, S. (2007). The interplay between cognitive and motivational variables in a problem-based learning environment. Learning and Individual Differences, 17, 291-297.

Arias-Gundín, O, Fidalgo, R. y García, J. N. (2008). El desarrollo de las competencias transversales en Magisterio mediante el APB y el Método de Caso. Revista de Investigación Educativa, 26, 431-444.
Baños, J. y Pérez, J. (2005). Cómo fomentar las competencias transversales en los estudios de Ciencias de la Salud: una propuesta de actividades. Educación médica, 8, 216-225.

Barca, A., Seijas, S., Brenlla, J.C. y Santamaría, S. (2000). La escala CEPEA (Cuestionario de evaluación de procesos de estudio y aprendizaje): un instrumento para la evaluación de los procesos de estudio y aprendizaje en el alumnado universitario. Revista galego-portuguesa de psicoloxía e educación: revista de estudios e investigación en psicología y educación, 5, 325-341.

Bernardo, A., Núñez, J., González-Pienda, J., Rosario, P., Álvarez, L., González, P., Valle, A., Rodríguez, S., Cerezo, R., Álvarez, D. y Rodríguez, C. (2009). Estilos intelectuales y rendimiento académico: una perspectiva evolutiva. Psicothema, 21, 555-561.

Camarero, F., Martín, F. y Herrero, J. (2000). Estilos y estrategias de aprendizaje en estudiantes universitarios. Psicothema, 12, 615-622.

Camina, A. y Salvador, M.I. (2007). Condicionantes y características de los estudiantes que inician magisterio. Estudio descriptivo y comparativo entre especialidades. Tendencias pedagógicas, 12, 245-262.

Cano, F. y Justicia, F.(2003). Factores académicos, estrategias y estilos de aprendizaje. Revista de Psicología general y aplicada, 46, 89-99.

Chapman, D. M. y Calhoun, J. G. (2006). Validation of learning style measures: implications for medical education practice. Medical Education, 40, 576-583.

De la Fuente, J. (2002). Perspectivas recientes en el estudio de la motivación: la teoría de la orientación de la meta. Escritos de Psicología, 6, 72-84.

Demirbas, O. y Demirkan, H (2007). Learning styles of design students and the relationship of academic performance and gender in design education. Learning and Instruction, 17, 345-359.

Díez, M. C., Pacheco, D. I., García, J. N., Mnez-Cocó, B., Robledo, P., Álvarez, M. L., Carbonero, M. A., Román, J. M., Caño, M. y Monjas, I. (2009). Percepción de los estudiantes universitarios de educación respecto al uso de metodologías docentes y el desarrollo de competencias ante la adaptación al EEES: datos de la Universidad de Valladolid. Aula Abierta, 37, 45-56.

English, L., Luckett, P. y Mladenovic, R. (2004). Encouraging a deep approach to learning through curriculum design. Accounting Education, 13, 461-488.

Farkas, R. D. (2003). Effects of traditional versus learningstyles instructional methods on middle school students. The Journal of Educational Research, 97, 42-51.

Felder, R. M., Felder, G. N. y Dietz, E. J. (2002). The effects of personality type on engineering student performance and attitudes. Journal of Engineering Education, 91, 3-17.

Fernández, M., García, J. N., de Caso, A. M., Fidalgo, R. y Arias-Gundín, O. (2006). El aprendizaje basado en prob- 
lemas: revisión de estudios empíricos internacionales. Revista de Educación, 341, 397-418.

Fidalgo, R. y García, J. N. (2008). Las Directrices del Espacio Europeo de Educación Superior en el Marco Legislativo del Sistema Universitario Español. Aula Abierta, 35, 35-48.

García, B. (2005). Estudio de los enfoques de aprendizajes en estudiantes de Magisterio y Psicopedagogía. Revista de investigación psicoeducativa psicopedagógica, 6-3,109126.

García, J. N. (2002). El Aprendizaje Basado en Problemas: ilustración de un modelo de aplicaciones en Psicopedagogía. Cultura y Educación, 14, 1-15.

García, J. N., de Caso, A. M., Fidalgo, R. y Arias-Gundín, O. (2005). La evaluación de prácticas Universitarias y su aplicación en un enfoque innovador. Revista de Educación. 337, 295-325.

García, J. N., Marbán, J, M., de Caso, A. M., Arias-Gundín, O., González, L., Martínez-Cocó, B., Rodríguez, C., Pacheco, D. I., Robledo, P., Díez, C. y Álvarez, L. (2008). Plan de innovación metodológica para la adaptación al EEES centrada en competencias: El uso del aprendizaje basado en problemas y el estudio de casos con la implementación de diversas herramientas activas. Actas V Congreso Internacional de Psicología y Educación: Los retos del futuro (pp. 2703-2709). Oviedo: Ediciones de la Universidad de Oviedo.

Gil, P., Contreras, O., Pastor, J., Gómez, I., González, S., García, L., de Moya, M.V. y López, A. (2007). Estilos de aprendizaje de los estudiantes de magisterio: especial consideración de los alumnos de educación física. Revista de curriculum y formación del profesorado, 11, 2-19.

Gómez, M. (2003). Identificación de los estilos de aprendizaje predominantes en estudiantes de magisterio de la Facultad de Ciencias de la Educación de la Universidad de Cádiz. Revista Electrónica Interuniversitaria de Formación del Profesorado, 6, 1-4.

Gómez, E., García, M.G. y Villalba, G. (2005). Una experiencia con el uso del portafolio del alumno en una asignatura de primer curso. En XI Jornadas de la enseñanza de universitaria de la informática, Madrid, España.

González, V. (2002). ¿Qué significa ser un profesional competente? Reflexiones desde una perspectiva psicológica. Revista Iberoamericana de educación, 22, 45-53.

González, G., Castro, A. y González, F. (2008). Perfiles aptitudinales, estilos de pensamiento y rendimiento académico. Facultad de Psicología - UBA// secretaría de investigaciones / anuario de investigaciones, 15, 33-41.

González-Pienda, J.A., Núñez, J.C., González-Pumariega, S., Álvarez, L., Roces, C., González, P., Bernardo, A., Valle, A., Cabanach, R.G., Rodríguez, S. y Rosario, P. (2004). Tinking styles: Analisis of its structural validity using adolescents' responses to the Thinking Styles Inventory. Psicothema, 16, 139-148.
Hur, Y. y Kim, S. (2007). Different outcomes of active and reflective students in problem-based learning. Medical Teacher, 29, 18-21.

Justicia, F. (2000). El comportamiento experto. En D. González, J.A. Amezcua, y F. Peñafiel (Coords.). El psicopedagogo en la organización y gestión de programas de formación (pp.89-103). Granada: GEU.

Li, Y.-S., Chen, P.-S. y Tsai, S.-J. (2008). A comparison of the learning styles among different nursing programs in Taiwan: implications for nursing education. Nurse Education Today, 28, 70-76.

Martín, F. y Camarero, F. (2001). Diferencias de género en los procesos de aprendizaje de los universitarios. Psicothema, 13, 598-604.

Martínez, M. y Crespo, E. (2008). Una aproximación a los factores que influyen en la motivación del alumnado de filología inglesa y traducción e interpretación. VI Jornadas de redes de investigación en docencia universitaria. Alicante, España.

Martínez-Cocó, B., García, J. N., Robledo, P., Díez, C., Álvarez, M. L., Marbán, J. M., De Caso, Fidalgo, R., Arias-Gundín, O., Pacheco, D. y Rodríguez, C. (2008). Valoración docente de las metodologías activas: un aspecto clave en el proceso de convergencia europea. Aula Abierta, 35, 49-62.

McDonough, J. P. y Osterbrink, J. (2005). Learning styles: an issue in clinical education? AANA Journal, 73, 89-93.

Rayner, S. y Riding, R. (1997). Towards a categorization of cognitive styles and learning styles. Educational Psychology, 17, 5-27.

Riding, R. y Rayner, S. (2002). Cognitive Styles and Learning Strategies. London: David Fulton Publishers.

Robledo, P., Marbán, J. y García, J. (2008). Experiencia piloto de innovación educativa en la asignatura Psicología de la educación y del desarrollo en edad escolar: utilización del portafolio y las tecnologías de la información en el proceso de adaptación al EEES. I Congreso sobre los títulos de educación en el marco del EEES. Málaga, España.

Rodríguez, M. L. (2005). Las demandas sociales y el mercado laboral ante la integración europea del conocimiento universitario. Cuadernos de integración europea, 2, 54-68.

Salim, R. (2006). El cuestionario CEPEA: herramienta de evaluación de Enfoques de aprendizaje en estudiantes universitarios. Revista Iberoamericana de Educación Número 36/4. http://www.rieoei.org/investigacion/1060Salim.PDF> [Consulta: julio 2009].

Sternberg, R.J., Castejón J.L. y Bermejo, M.R. (1999). Estilo intelectual y rendimiento académico. Revista de Investigación Educativa, 17, 33-46.

Sternberg, R.J. y Grigorenko, E.L. (1995). Styles of thinking in the school. European Journal for High Ability, 6, 201-219.

Sternberg, R. J. y Wagner, R.K. (1991). MSG Thinking Styles Inventory (manual). Departamento de Psicología, Universidad de Yale. Adaptación de J. A. González Pienda, J.C 
PATRICIA ROBLEDO RAMÓN, JESÚS NICASIO GARCÍA SÁNCHEZ, CARMEN DÍEZ GONZÁLEZ, Mª LOURDES ÁLVAREZ FERNÁNDEZ, JOSEFINA MILAGROS MARBÁN PÉREZ, ANA Mª DE CASO FUENTES, RAQUEL FIDALGO REDONDO, OLGA ARIAS GUNDÍN, DEILIS IVONNE PACHECO SANZ

Núñez, S. González Pumariega, L. Álvarez, C. Roces, y M. S. García (1997). Universidad de Oviedo.

Struyven, K., Dochy, F. y Janssens, S. (2005). Students' perceptions about evaluation and assessment in higher education: a review. Assessment y Evaluation in Higher Education, 30, 325-341.

Tickle, S. (2001). What have we learnt about student learning? A review of the research on study approach and style. Kybernetes, 30, 955-969.
Zhang, L.F. (2004). Revisiting the predictive power of thinking styles for academic performance. The Journal of Psychology, 138, 351-370.

Zhang, L.F., y Sternberg, R.J. (2005). Thinking styles, abilities and academic achievement among Hong Kong university students. Educational Research Journal, 13, 41-62.

Fecha de recepción: 7 de julio de 2009

Fecha de aceptación: 7 de julio de 2010 